\title{
Breast Milk-Transmitted Cytomegalovirus Infection in Preterm Infants
}

\author{
Mi Hyeon Gang and Mea-young Chang \\ Department of Pediatrics, School of Medicine, Chungnam National University, Daejeon, Korea
}

\section{ABSTRACT}

Purpose: The purpose of this study is to describe the rate of cytomegalovirus (CMV) virolactia, and the prevalence of breast milk (BM)-transmitted postnatal CMV infection among premature infants after freeze-thawing (FT) and Holder pasteurization (HP) of breast milk.

Methods: This is a single-center, retrospective study of 312 infants born at less than 32 weeks of gestation, or with a birth weight less than 1,500 g from January 2013 to June 2017. All infants were screened for CMV-specific immunoglobulin (Ig) G and IgM at birth. Initial CMV specific polymerase chain reaction (PCR) and CMV culture were performed on mothers' BM and babies' urine within the first 21 days of life. FT and HP of $\mathrm{BM}$ was used to prevent the transmission of CMV. For the surveillance of postnatal CMV infection, CMV culture and CMV specific PCR of urine from babies were repeated one to two months after the initial screening. Screening for viremia and viruria was performed if postnatal CMV infection was suspected.

Results: Among $178 \mathrm{BM}$ samples obtained from mothers of CMV-IgG-seropositive infants, 80 (44.9\%) were CMV PCR positive. CMV deoxyribonucleic acid (DNA) was detected in five of the $22 \mathrm{BM}$ samples (22.7\%) obtained from the mothers of CMV-IgG seronegative infants. When CMV DNA load in BM was measured before and after HP, various results were shown. Sixty-three infants out of 232 (27.2\%) were evaluated for postnatal CMV infection and four infants out of $63(6.3 \%)$ were infected.

Conclusion: Interventions to prevent BM-transmitted CMV infection can reduce the chance of postnatal CMV infection, but not completely eliminate it.

Key Words: Cytomegalovirus, Infection, Human milk, Freeze-thawing, Holder pasteurization, Premature infant

\section{INTRODUCTION}

Cytomegalovirus (CMV) may be transmitted in utero as a result of maternal infection, and may also be acquired perinatally at delivery from exposure to maternal genital secretions, or through blood transfusions (BTs) or infected breast milk (BM $)^{1)}$.
Received: 25 July 2017

Revised: 28 December 2017

Accepted: 21 January 2018

Correspondence to: Mea-young Chang Department of Pediatrics, School of Medicine, Chungnam National University, 282 Munhwa-ro, Jung-gu, Daejeon 35015, Korea

Tel: +82-42-280-7253

Fax: +82-42-255-3158

E-mail:mychang@cnuh.co.kr

Copyright(c)

By Korean Society of Neonatology.

All right reserved.

This is an Open-Access article distributed under the terms of the Creative Commons Attribution Non-Commercial License (http://creativecommons.org/licenses/ by-nc/4.0), which permits unrestricted non-commercial use, distribution, and reproduction in any medium, provided the original work is properly cited. 
Postnatal CMV infection has not received much attention in the past, because it was thought to have no significant morbidity. However, as studies on postnatal CMV infection with serious clinical symptoms, especially in preterm infants, have been reported, interest in the seriousness of postnatal CMV infection has increased.

BT practices in Korea do not involve the routine testing of donor blood for CMV antibodies ${ }^{2)}$. The risk of CMV infection from BTs is related to the volume of transfused blood, number of donors, and elevated complement fixation titers to CMV in the donor blood. The use of leukodepleted and/or CMV-negative blood eliminates the risk of CMV acquisition via this route ${ }^{3)}$.

The majority of CMV-seropositive women shed the virus in their $\mathrm{BM}$. There is considerable evidence showing that the shedding of the virus into $\mathrm{BM}$ is the main source of postnatal CMV infection in infants ${ }^{4)}$. Because more than $80 \%$ of South Korean women have CMV antibodies by childbearing age ${ }^{5}$, the transmission rate of CMV from mothers to preterm infants by breastfeeding is assumed to be high.

Symptomatic CMV infections do not usually occur in fullterm infants because of the transmission of protective maternal antibodies, which occurs mostly in the third trimester, and the infants' more mature immune system ${ }^{1,6)}$. Infants born at less than 32 weeks of gestation or with very low birth weight (VLBW; birth weight $<1,500 \mathrm{~g}$ ) may be at a higher risk of developing symptomatic postnatal CMV disease ${ }^{1)}$.

It is not possible to control the exposure to CMV in maternal genital secretions during vaginal delivery. Transfusion-transmitted CMV infection can be reduced by the aforementioned methods. Thus, prevention of transmission of CMV to preterm infants through $\mathrm{BM}$ is an important issue, and it is prudent to try to prevent postnatal CMV transmission to preterm infants by giving them BM with no infectivity.

Aims of this study are: (1) to estimate CMV seroprevalence among pregnant women by investigating the positive rates of CMV-immunoglobulin (Ig) G in enrolled infants, (2) to determine the rate of CMV detection in BM from mothers of preterm infants, and CMV viral load in their BM before and after Holder pasteurization (HP), and (3) to determine the incidence of postnatally acquired CMV infection via BM, after freeze-thawing (FT) and $\mathrm{HP}$, among preterm infants during their hospitalization in our center.

\section{MATERIALS AND METHODS}

In this single-center retrospective study, one physician reviewed the medical records of the study subjects, and collected information on demographics, gestational age (GA), birth weight, delivery method, laboratory data associated with CMV infection, maternal ethnic ancestry, and transfusion history.

Premature babies with a GA of less than 32 weeks, or VLBW infants who were admitted between January 1, 2013 and June 30, 2017 to the Chungnam National University Hospital were included in the study.

Infants were excluded if they showed evidence of congenital CMV infection, defined as a polymerase chain reaction (PCR)positive viral deoxyribonucleic acid (DNA) in urine (DNAuria) or culture-positive urine (viruria) within the first 3 weeks of life ${ }^{1)}$.

Preterm infants born at less than 32 weeks of gestation, or VLBW infants were screened for both CMV-specific IgG and IgM in their blood. We used an enzyme-linked immunosorbent assay (ELISA) to detect CMV-IgG and IgM (Instrument: Chorus, Diesse Diagnostica Senese, Milano, Italy). CMV PCR and culture were performed on the BM samples from mothers and urine specimens from infants collected in a urine bag within the first 21 days of life (DOLs). To monitor the presence or absence of postnatal CMV infection, CMV PCR and culture of urine were repeated 1 to 2 months after the initial screening. Postnatal CMV infection was defined as DNAuria and/or viruria 3 weeks after birth $^{7 \text { ) }}$. If symptomatic CMV infection was suspected, urine and whole blood were tested by CMV PCR and culture. CMV DNA was extracted from the collected clinical samples using ExiPrep ${ }^{\mathrm{TM}}$ Dx Viral DNA Kit (Bioneer Corp., Daejeon, Korea) within 1 day, according to the instructions provided by the manufacturers. AccuPower ${ }^{\circledR}$ CMV Quantitative PCR Kit (Bioneer Corp.) was used for virus testing by real-time PCR. The CMV culture method is as follows: Round cover slips were placed in each well of a 24well plate, and each well was seeded with planting medium and MRC- 5 cells. The plate was placed in a $\mathrm{CO}_{2}$ incubator for 72 hours. After removing the planting medium, RM-02 Refeed medium was added to the wells. The pre-treated specimens were inoculated into each well, centrifuged at $30^{\circ} \mathrm{C}$ for 50 minutes, and cultured for 3 days at $37^{\circ} \mathrm{C}$ in a $\mathrm{CO}_{2}$ incubator. The specimens were fluorescently stained and examined by fluorescence microscopy.

Additionally, the viral load in $11 \mathrm{BM}$ samples was measured before and after HP if BM of the mothers of premature babies born at less than 32 weeks of gestation or of VLBW infants was 
CMV DNA positive. CMV DNA loads in positive BM samples were analyzed using R version 3.2.5 (R Core Team 2016, Vienna, Austria). The values less than the limit of quantitation (LOQ) were substituted with a constant value of the LOQ divided by the square root of $2^{8)}$.

In our center, transfusion-associated virus transmission was prevented by exclusive use of $\gamma$-irradiated blood products of unknown CMV status after leukodepletion by filtration. Breastfed preterm infants at high risk of postnatal CMV infection were exclusively fed with FT and pasteurized BM until 8 weeks of age, regardless of postmenstrual age (PMA). FT and HP were always performed together in sequence. FT involves freezing human milk at $-20^{\circ} \mathrm{C}$ for several days and thawing just prior to each feeding. $\mathrm{HP}$ involves heating $\mathrm{BM}$ at $62.5^{\circ} \mathrm{C}$ for 30 minutes. A self-designed water bath was used to maintain the water temperature consistently at $62.5^{\circ} \mathrm{C}$ for 30 minutes. Inadequate amounts of BM were supplemented with preterm formula milk. All the study patients except one were fed more than $50 \mathrm{cc} / \mathrm{kg} /$ day of BM for more than a week. One patient who died on the 25th DOL was hemodynamically unstable and breastfed for 3 days.

The study protocol was approved by the Institutional Review Board of Chungnam National University Hospital, Daejeon, Korea (IRB No. 2017-05-009).

The continuous variables of BM samples between two groups were compared using Welch's $t$-test. Statistical significance was defined as a $P$-value of less than 0.05 .

\section{RESULTS}

Among the 312 patients born at less than 32 weeks of gestation,

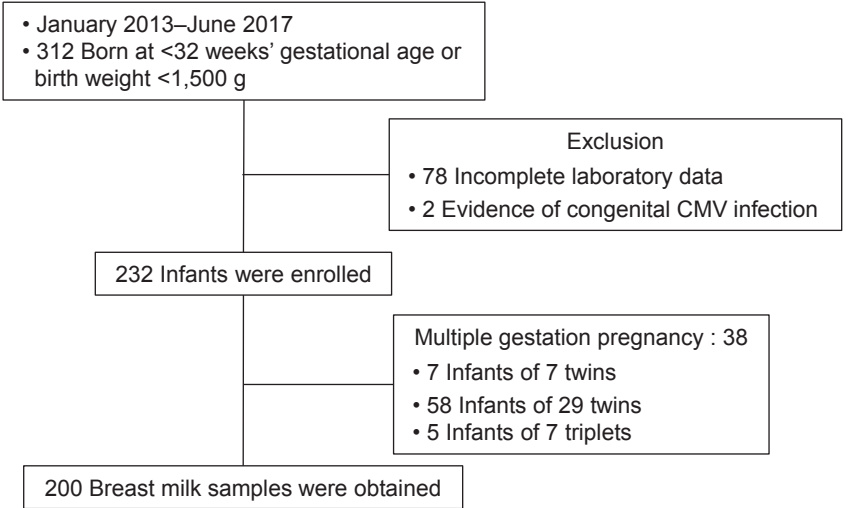

Figure 1. Diagram showing the enrollment of study patients. Abbreviation: CMV, cytomegalovirus. or with a birth weight of less than 1,500 g, 80 were excluded due to incomplete laboratory data and evidence of congenital CMV infection, and a total of 232 infants were enrolled in the study. Two hundred BM samples were obtained due to multiple gestation pregnancy (Figure 1).

Table 1 shows the characteristics of the subjects. The ratio of infants delivered through cesarean section was as high as $81.0 \%$. Most mothers were Korean. The rate of CMV-IgG seropositivity among the 232 enrolled patients was $90.1 \%$.

CMV PCR and culture were performed on the mothers' BM at a mean infant age of 5.5 days. Eighty (44.9\%) out of 178 BM samples from mothers of CMV-IgG seropositive infants, and five (22.7\%) out of $22 \mathrm{BM}$ samples from mothers of CMV-IgG seronegative infants were CMV PCR positive. Conversely, among 85 BM samples that were CMV PCR positive, $80 \mathrm{BM}$ samples (94.1\%) were obtained from mothers of CMV-IgG seropositive infants. Only three out of 85 BM samples positive for CMV PCR were CMV culture positive (Figure 2).

Excluding two extraordinary outliers (more than 100,000 copies/ $\mathrm{mL}$ ) from the sample, maternal milk of CMV-IgG-seropositive infants had higher median viral loads than that of CMV-IgG seronegative infants (median, 3,346.7 vs. 409.0, $P=0.001$ ) (Figure 3).

CMV DNA loads in $11 \mathrm{BM}$ samples before and after HP are shown in Figure 4. It showed varying results; some decreased and some remained constant. Moreover, the majority of CMV DNA loads after HP were above the LOQ.

Sixty-three infants out of $232(27.2 \%)$ were examined for the surveillance of postnatal CMV infection, and four infants out

Table 1. Characteristics of infants $(n=232)$

\begin{tabular}{lc}
\hline Variable & Value \\
\hline Gestational age (wk) & $30.0(22.9-36)$ \\
Birth weight (g) & $1,300(380-2,120)$ \\
Female sex & $102(44.0)$ \\
Cesarean section & $188(81.0)$ \\
Maternal ethnic ancestry & \\
Korea & 194 \\
China & 1 \\
Mongolia & 1 \\
Philippines & 2 \\
Vietnam & 2 \\
Transfusion of cellular blood components & $130(56.0)$ \\
CMV-IgG seropositivity & $209(90.1)$
\end{tabular}

Values are expressed as median (range) or number (\%). Abbreviation: CMV-IgG, cytomegalovirus-immunoglobulin G. 


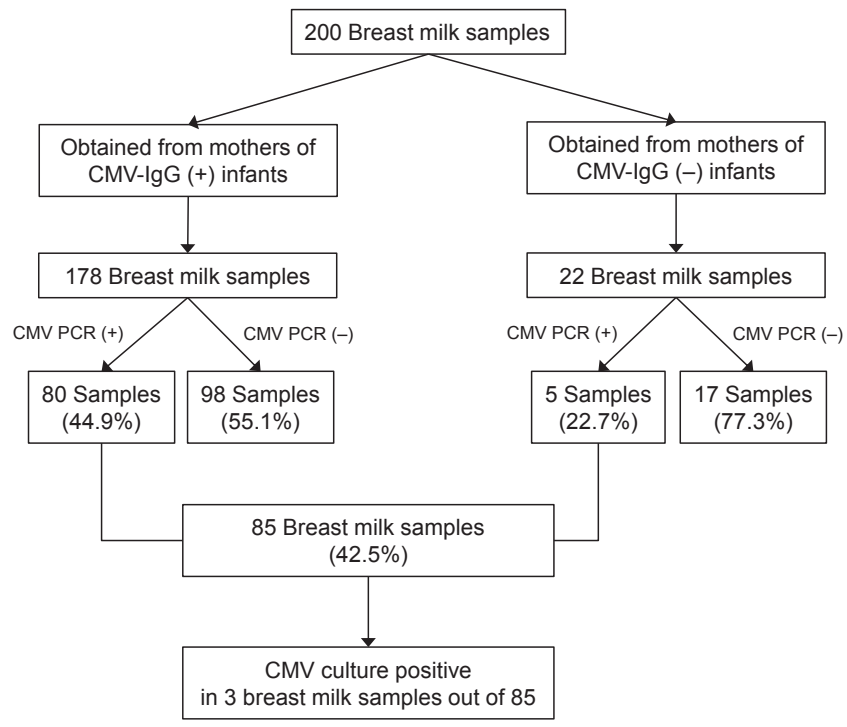

Figure 2. Cytomegalovirus (CMV) deoxyribonucleic acid (DNA) polymerase chain reaction (PCR) analysis of breast milk. Abbreviation: IgG, immunoglobulin G.

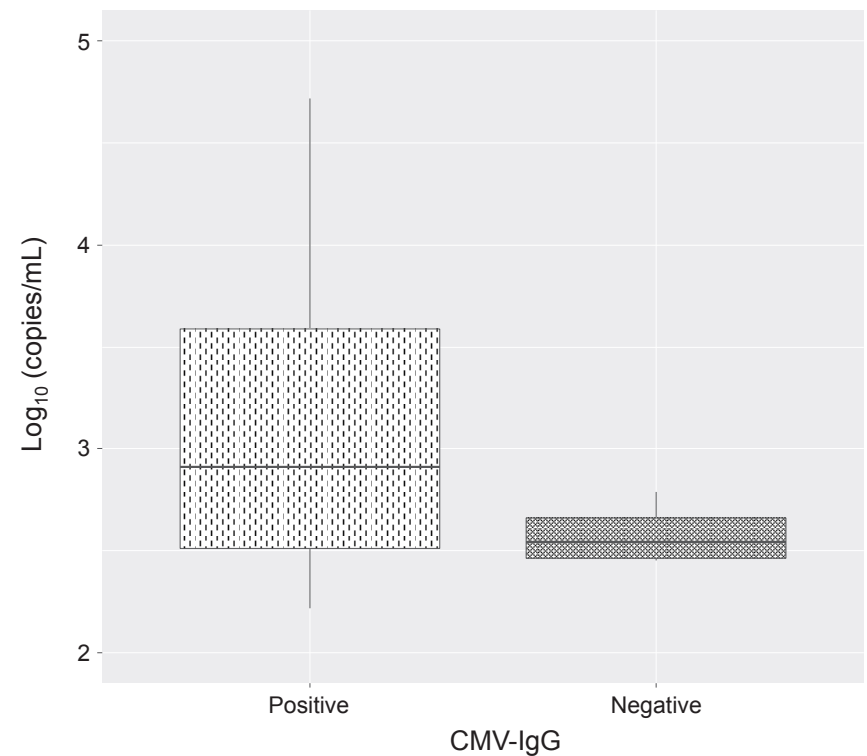

Figure 3. Log-transformed cytomegalovirus (CMV) deoxyribonucleic acid (DNA) copy numbers in positive breast milk samples. Maternal milk of CMV-immunoglobulin G (IgG)-seropositive infants at birth had higher mean viral loads than that of CMV-IgG seronegative infants.

of the 63 studied infants (6.3\%) were infected. Three of the four infected infants were born to mothers who shed CMV in milk. All four infants were born via caesarean section, and one out of four babies did not receive any blood transfusions. Three of the affected premature babies were asymptomatic. One baby, who had a birth weight of $1,110 \mathrm{~g}$ and was born at $272 / 7$ weeks of

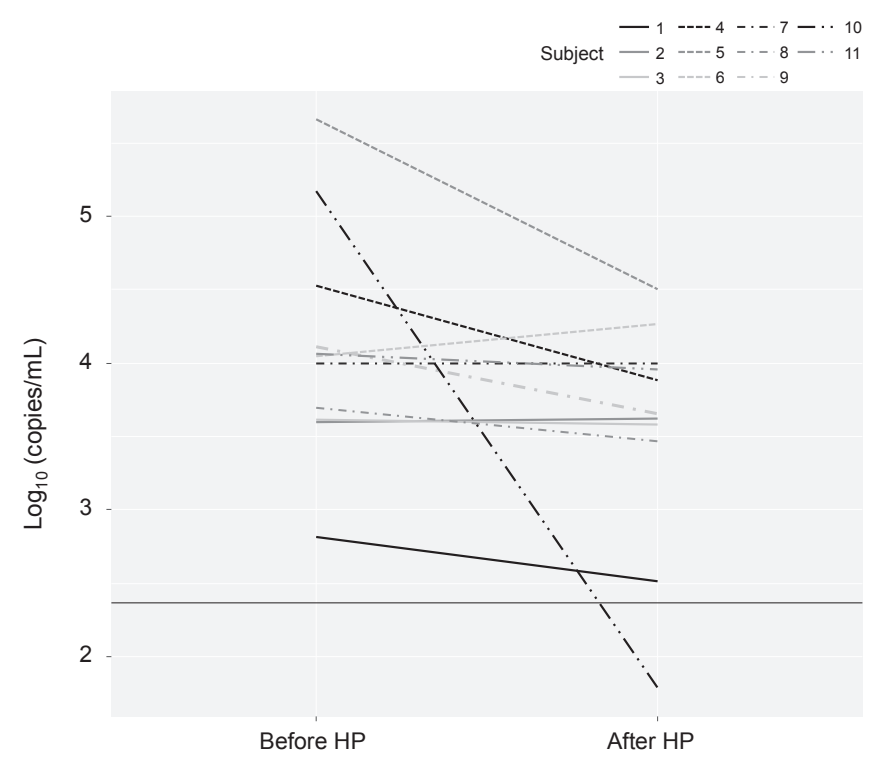

Figure 4. Log-transformed cytomegalovirus (CMV) deoxyribonucleic acid (DNA) copy numbers in breast milk before and after Holder pasteurization (HP). CMV DNA loads decreased after HP in some cases and were not significantly changed in other cases. The black line represents the limit of quantitation (LOQ) value. Majority of the values were above the LOQ.

gestation, developed sepsis-like syndrome (SLS).

\section{DISCUSSION}

In our retrospective study, postnatal CMV infection was diagnosed in only four infants (4/232 of enrolled infants [1.7\%] and 4/63 of infants [6.3\%] examined for the surveillance of postnatal CMV infection) despite the high rate of viral shedding into BM, and it is presumed that FT and HP might reduce the risk of BMtransmitted CMV infection. Kurath et al. ${ }^{9)}$ reported that CMV-IgG detection in breast milk in the range $67 \%$ to $97.2 \%$ (median, $80 \%$ ), the CMV transmission rate varied in a range of $5.6 \%$ to $58.6 \%$ (median, 20\%) in preterm infants fed with untreated BM, and symptomatic disease occurred in $0 \%$ to $34.5 \%$ (mean, $3.7 \%$ ) of the cases.

Postnatal CMV infection can be diagnosed by the detection of CMV using culture or PCR of blood, urine, cerebrospinal fluid, or respiratory secretions after 2 to 3 weeks of life ${ }^{1,7,10)}$. Demonstration of CMV-specific IgM in cord blood or in the infant's blood within the first 2 to 3 weeks of life suggests congenital CMV infection ${ }^{7,10)}$. However, only $70 \%$ of congenitally infected neonates have IgM antibodies at birth ${ }^{11)}$. The presence of CMV-specific IgG indicates 
its passive transfer from the mother or a congenital infection, and does not diagnose neonatal infection ${ }^{11)}$. Therefore, while no test was performed on the mother's CMV status, we assumed that CMV-IgG positivity in the babies indicates CMV infection in their mothers, and also a possibility of CMV virolactia. We have performed CMV PCR and culture on the babies' urine within the first 21 DOL to exclude congenital CMV infection.

Traditionally, CMV infection have been diagnosed by the isolation of the virus from the urine or saliva by virus culture methods. Virus culture may take a long time to yield results, and has an advantage over PCR in terms of specificity ${ }^{11)}$. The shell-vial culture assay uses a monoclonal antibody to detect early CMV antigen after the specimen is incubated in tissue culture for 24 to 48 hours $^{12)}$. However, set-up costs for a virus culture laboratory are expensive $^{13)}$. On the other hand, the identification of CMV DNA by PCR in urine, blood, saliva, cerebrospinal fluid, and bronchoalveolar lavage fluid, or tissue is rapid, highly sensitive, and provides viral load measurements ${ }^{14,15}$. The negative predictive value of PCR is high ${ }^{15)}$.

Maternal CMV shedding in BM of seropositive mothers can be detected in colostrum and ends about 3 months after birth ${ }^{16}$. Yasuda et al. ${ }^{17)}$ showed that 21 of the 24 seropositive mothers (87.5\%) had detectable CMV DNA in their BM during their study period and most of the BM samples became positive for CMV DNA 2 weeks after delivery. According to several other studies, rates of CMV virolactia among seropositive women have been reported to be between $6.2 \%$ and $80 \%{ }^{18,19)}$. In our study, 85 of the $200 \mathrm{BM}$ samples (42.5\%) and 80 of the total $178 \mathrm{BM}$ samples (44.9\%) from mothers of CMV-IgG seropositive infants were CMV PCR positive. Compared to previous studies, the rate of CMV virolactia in the mothers in our study was intermediate, which can be ascribed to the fact that the test was performed right after delivery, before the rise of CMV load in BM. It was also noticed that some BM samples from mothers of CMV-IgG-seronegative infants were CMV PCR positive. There may be a number of reasons for this: (1) It might be possible that the GA at birth was so low that the maternal antibodies were not transferred to the baby. Therefore, there is a possibility that those low-GA babies, despite the low milk CMV loads, may be more susceptible to severe, symptomatic diseases. (2) False positives and false negatives may have altered the results.

In our center, CMV PCR and culture were performed simultaneously for all specimens. Eighty-five of the total $200 \mathrm{BM}$ samples (42.5\%) were CMV PCR positive. However, the culture positivity rate was low (three of 85 PCR-positive BM samples [3.5\%]) compared to a culture sensitivity of $8 \%$, as reported by Weinberg et al. ${ }^{20)}$, even though the CMV culture was conducted at an accredited laboratory after collecting and transporting the specimens according to the guidelines. There is no clear explanation for these discrepancies between the PCR and culture results; further studies may be suggested.

We demonstrated that viral loads were higher in maternal milk of CMV-IgG-seropositive infants than in that of CMV-IgG seronegative infants. van der Strate et al. ${ }^{21)}$ showed that viral loads in the milk whey of CMV-transmitting mothers were higher than those of nontransmitting mothers; transmission of CMV to newborns was observed only above the threshold. On the other hand, Josephson et al. ${ }^{10)}$ reported that they could not identify a viral load cutoff below which BM-transmitted CMV infections did not occur. Although the viral load cut-off below which BM-transmitted CMV infections do not occur is yet to be determined, our results may be used to preferentially select infants at a high risk of BMtransmitted CMV infections in cases where maternal CMV status, occurrence of CMV shedding in BM, or viral load is unknown.

It was estimated that the chance of exposure to genital secretions in the enrolled infants was relatively low because the rate of cesarean section was high. The use of leukodepleted cellular blood products eliminated the risk of transfusion-acquired CMV infection. Previous studies have shown that the possibility of CMV infection through transfusion is very low when using filters ${ }^{22)}$. In this study, all four infants who were positive for urine CMV PCR were born via caesarean section, one out of four babies did not receive any blood transfusions, and three out of four babies received one or more transfusions of irradiated and leukodepleted cellular blood products; hence, the possibility of transmission by maternal genital secretion or BT was ruled out.

Since several strategies to prevent the transmission of CMV to preterm infants through BM have been suggested, we selected a few and applied them to our unit's policy. In our hospital, premature infants were fed FT and pasteurized BM until 8 weeks of life because postnatal CMV infection within 8 weeks of postnatal age can cause serious complications ${ }^{23)}$, and the CMV DNA copy numbers in BM peak at 4 to 6 weeks and decrease afterward ${ }^{17,24)}$. FT does not completely eliminate the virus, but is less harmful to the protective constituents in milk ${ }^{25-28)}$. According to Lanzieri et al. ${ }^{1)}$, among the infants who were fed frozen BM, 13\% (95\% confidence interval [CI], 7\% to $24 \%$ ) acquired CMV infection, $7 \%$ (95\% CI, 3\% to $14 \%$ ) developed CMV-related symptoms, and 5\% 
(95\% CI, $2 \%$ to $12 \%$ ) developed CMV-SLS. Other studies reported a CMV transmission rate of $2.5 \%$ to $15 \%$ when FT BM is used ${ }^{29-}$ 31). While HP is highly effective at removing viable CMV, it may inactivate biologically active components such as Igs, enzymes, hormones, growth factors, cytokines, and heat-labile vitamins ${ }^{6,32)}$. Hamprecht et al. ${ }^{33)}$ reported that HP did not reduce the viral DNA load significantly, but eliminated CMV infectivity. Postnatally acquired CMV infection rates in premature infants fed pasteurized $\mathrm{BM}$ have been variously reported in the literature; Yoo et al. ${ }^{34)}$ reported an infection rate of $0 \%$, and Stock et al. ${ }^{35)}$ reported $4.2 \%$. The reduction rate of CMV infection via mother's milk through $\mathrm{HP}$ is still unknown, and more research is needed. In addition, Figure 4 shows that the protective effect of HP on BM-transmitted CMV infection is doubtful. When CMV DNA load in BM was measured before and after HP, varying results were obtained. While some decreased, others remained constant, with the majority of CMV DNA loads after HP being above the LOQ. Moreover, it should be acknowledged that neither do values below the LOQ confirm negative infectivity nor does PCR positivity directly demonstrate positive infectivity. Nevertheless, it is only reasonable to take heed of the positive conversion of CMV in infants fed BM subjected to FT and HP, as it may indicate postnatal CMV infection. Two promising techniques, microwave irradiation and ultraviolet- $\mathrm{C}$ irradiation, have been recently introduced to eradicate the virus, but further studies are needed to prove their efficiency ${ }^{36,37)}$.

Postnatal infection does not always mean disease, but in premature infants, it can cause severe clinical symptoms due to their immunocompromised status and deficiency of maternal CMV-IgG antibodies $^{24,38)}$. In symptomatic postnatal CMV disease, a number of clinical symptoms and signs including pneumonitis, hepatosplenomegaly, lymphadenopathy, enteritis, aseptic meningitis, and SLS can develop, and all cultures (blood, urine, tracheal aspirates, and stool) for bacterial pathogens obtained before initiation of antibiotic treatment are found to be sterile. CMV is detected by culture or PCR of blood, urine, cerebrospinal fluid, or respiratory secretions after DOL $21^{18,39)}$. Okulu et al. ${ }^{40)}$ reported a case of an extremely low-birth-weight infant with severe postnatal CMV infection presenting with hepatitis and pneumonitis. In a case report published by Tran et al. ${ }^{41)}$, a 48-day-old infant born at $251 / 7$ weeks of GA without symptoms of perinatally acquired CMV developed fulminant necrotizing enterocolitis on DOL 48 and systemic CMV infection was diagnosed at autopsy; he died despite aggressive medical management. In our case, a baby born at $272 / 7$ weeks of GA developed SLS even though he was not exposed to genital secretions at birth because he was born via cesarean section. A leukodepletion filter was used for BT, and he was fed FT and pasteurized BM. His postnatal CMV infection was confirmed by testing urine, tracheal secretion, and whole blood for CMV DNA with PCR, which revealed high viral loads. This indicates that breakthrough cases may occur even with the use of several prevention strategies. At present, guidelines for the treatment of congenital CMV infection have been suggested ${ }^{42)}$. But in cases of perinatally or postnatally acquired symptomatic CMV infection, there is no consensus on the management, although the use of antiviral treatment in severe and symptomatic cases have been reported $^{39,40)}$.

Long-term outcomes in preterm infants after postnatal CMV infection are controversial. Kelly et al. ${ }^{7)}$ demonstrated that postnatal CMV infection was associated with an increased risk of bronchopulmonary dysplasia at 36 weeks of PMA in VLBW infants. Some follow-up studies indicated poor neurocognitive outcome in adolescents born preterm with postnatal CMV infection $^{43,44)}$. In contrast, other studies showed that preterm infants might not have an increased risk for neurological sequelae after CMV postnatal infection, ${ }^{9,45)}$.

Considering the clinical significance of symptomatic postnatal CMV infection in preterm infants, some people might think that it is better to withhold BM feeding in preterm infants until 34 weeks of PMA ${ }^{10)}$. However, as the incidence of symptomatic $\mathrm{CMV}$ infection during the entire study period was not high, $\mathrm{BM}$ preparations to decrease CMV transmission in high risk infants, along with prompt workup and treatment in suspected patients can be an appropriate method of maximizing the advantages of BM feeding.

Our study is limited by its retrospective nature. Maternal CMV infection could not be determined exactly because the maternal CMV serostatus was not examined in our hospital. Enrolled infants were not tested for CMV culture (e.g., nasal swab) immediately after birth to confirm whether they had been exposed to maternal genital secretions. Follow-up CMV testing of the asymptomatic baby was not routinely performed as planned, and hence, postnatally acquired CMV infection could be underestimated.

In summary, despite the relatively high rate of viral shedding into BM, postnatal CMV infection was diagnosed in $1.7 \%$ of the total infants enrolled, and $6.3 \%$ of the infants examined for the surveillance of postnatal CMV infection after FT and HP. It is assumed that these interventions to prevent BM-transmitted CMV infection can reduce the chance of symptomatic postnatal CMV 
infection, but not completely eliminate it. Therefore, physicians must be reminded of the possibility and risks of breakthrough infection even in FT and pasteurized BM-fed infants. More data is needed in order to construct an effective prevention strategy against CMV infection, and to form a consensus guideline concerning the diagnosis and treatment of postnatal CMV infection in preterm infants. Further research on the long-term outcome of postnatal CMV infection may also be suggested.

\section{CONFLICT OF INTEREST}

No potential conflict of interest relevant to this article was reported.

\section{REFERENCES}

1) Lanzieri TM, Dollard SC, Josephson CD, Schmid DS, Bialek SR. Breast milk-acquired cytomegalovirus infection and disease in VLBW and premature infants. Pediatrics 2013;131:e1937-45.

2) Kim AR, Lee YK, Kim KA, Chu YK, Baik BY, Kim ES, et al. Transfusion-related cytomegalovirus infection among very low birth weight infants in an endemic area. J Korean Med Sci 2006; 21:5-10.

3) Swanson EC, Schleiss MR. Congenital cytomegalovirus infection: new prospects for prevention and therapy. Pediatr Clin North Am 2013;60:335-49.

4) Ehlinger EP, Webster EM, Kang HH, Cangialose A, Simmons AC, Barbas KH, et al. Maternal cytomegalovirus-specific immune responses and symptomatic postnatal cytomegalovirus transmission in very low-birth-weight preterm infants. J Infect Dis 2011;204:1672-82.

5) Sohn YM, Park KI, Lee C, Han DG, Lee WY. Congenital cytomegalovirus infection in Korean population with very high prevalence of maternal immunity. J Korean Med Sci 1992;7:47-51.

6) Bryant P, Morley C, Garland S, Curtis N. Cytomegalovirus transmission from breast milk in premature babies: does it matter? Arch Dis Child Fetal Neonatal Ed 2002;87:F75-7.

7) Kelly MS, Benjamin DK, Puopolo KM, Laughon MM, Clark RH, Mukhopadhyay S, et al. Postnatal cytomegalovirus infection and the risk for bronchopulmonary dysplasia. JAMA Pediatr 2015;169:e153785.

8) Succop PA, ClarkS, Chen M, Galke W. Imputation of data values that are less than a detection limit. J Occup Environ Hyg 2004; 1:436-41.

9) Kurath S, Halwachs-Baumann G, Muller W, Resch B. Trans- mission of cytomegalovirus via breast milk to the prematurely born infant: a systematic review. Clin Microbiol Infect 2010; 16:1172-8.

10) Josephson CD, Caliendo AM, Easley KA, Knezevic A, Shenvi $\mathrm{N}$, Hinkes MT, et al. Blood transfusion and breast milk transmission of cytomegalovirus in very low-birth-weight infants: a prospective cohort study. JAMA Pediatr 2014;168:1054-62.

11) Leung AK, Sauve RS, Davies HD. Congenital cytomegalovirus infection. J Natl Med Assoc 2003;95:213-8.

12) Stehel EK, Sanchez PJ. Cytomegalovirus infection in the fetus and neonate. Neoreviews 2005;6:e38-45.

13) Trincado DE, Rawlinson WD. Congenital and perinatal infections with cytomegalovirus. J Paediatr Child Health 2001;37:18792.

14) Malm G, Engman ML. Congenital cytomegalovirus infections. Semin Fetal Neonatal Med 2007;12:154-9.

15) Boeckh M. Complications, diagnosis, management, and prevention of CMV infections: current and future. Hematology Am Soc Hematol Educ Program 2011;2011:305-9.

16) Hamprecht K, Witzel S, Maschmann J, Dietz K, Baumeister A, Mikeler E, et al. Rapid detection and quantification of cell free cytomegalovirus by a high-speed centrifugation-based microculture assay: comparison to longitudinally analyzed viral DNA load and pp67 late transcript during lactation. J Clin Virol 2003;28:303-16.

17) Yasuda A, Kimura H, Hayakawa M, Ohshiro M, Kato Y, Matsuura $\mathrm{O}$, et al. Evaluation of cytomegalovirus infections transmitted via breast milk in preterm infants with a real-time polymerase chain reaction assay. Pediatrics 2003;111(6 Pt 1):1333-6.

18) Vochem M, Hamprecht K, Jahn G, Speer CP. Transmission of cytomegalovirus to preterm infants through breast milk. Pediatr Infect Dis J 1998;17:53-8.

19) Doctor S, Friedman S, Dunn MS, Asztalos EV, Wylie L, Mazzulli T, et al. Cytomegalovirus transmission to extremely lowbirthweight infants through breast milk. Acta Paediatr 2005; 94:53-8.

20) Weinberg A, Hodges TN, Li S, Cai G, Zamora MR. Comparison of PCR, antigenemia assay, and rapid blood culture for detection and prevention of cytomegalovirus disease after lung transplantation. J Clin Microbiol 2000;38:768-72.

21) van der Strate BW, Harmsen MC, Schafer P, Swart PJ, The TH, Jahn G, et al. Viral load in breast milk correlates with transmission of human cytomegalovirus to preterm neonates, but lactoferrin concentrations do not. Clin Diagn Lab Immunol 2001; 8:818-21.

22) Gilbert GL, Hayes K, Hudson IL, James J. Prevention of transfusion-acquired cytomegalovirus infection in infants by blood filtration to remove leucocytes. Neonatal Cytomegalovirus Infection Study Group. Lancet 1989;1:1228-31. 
23) Paryani SG, Yeager AS, Hosford-Dunn H, Johnson SJ, Malachowski N, Ariagno RL, et al. Sequelae of acquired cytomegalovirus infection in premature and sick term infants. J Pediatr 1985;107: 451-6.

24) Hamprecht K, Goelz R, Maschmann J. Breast milk and cytomegalovirus infection in preterm infants. Early Hum Dev 2005; 81:989-96

25) Liebhaber M, Lewiston NJ, Asquith MT, Olds-Arroyo L, Sunshine P. Alterations of lymphocytes and of antibody content of human milk after processing. J Pediatr 1977;91:897-900.

26) Welsh JK, Arsenakis M, Coelen RJ, May JT. Effect of antiviral lipids, heat, and freezing on the activity of viruses in human milk. J Infect Dis 1979;140:322-8.

27) Friis $H$, Andersen HK. Rate of inactivation of cytomegalovirus in raw banked milk during storage at -20 degrees $\mathrm{C}$ and pasteurisation. Br Med J (Clin Res Ed) 1982;285:1604-5.

28) Hosseini M, Esmaili HA, Abdoli Oskouei S, Gojazadeh M, MokariYamchi Z, Layegh V, et al. Evaluation of the freeze-thawing method in reducing viral load of cytomegalovirus in breast milk of mothers of preterm infants. Breastfeed Med 2016;11:55760.

29) Jim WT, Shu CH, Chiu NC, Kao HA, Hung HY, Chang JH, et al. Transmission of cytomegalovirus from mothers to preterm infants by breast milk. Pediatr Infect Dis J 2004;23:848-51.

30) Chiavarini M, Bragetti P, Sensini A, Cenci E, Castronari R, Rossi MJ, et al. Breastfeeding and transmission of cytomegalovirus to preterm infants. Case report and kinetic of CMV-DNA in breast milk. Ital J Pediatr 2011;37:6.

31) Hayashi S, Kimura H, Oshiro M, Kato Y, Yasuda A, Suzuki C, et al. Transmission of cytomegalovirus via breast milkin extremely premature infants. J Perinatol 2011;31:440-5.

32) Andersson Y, Savman K, Blackberg L, Hernell O. Pasteurization of mother's own milk reduces fat absorption and growth in preterm infants. Acta Paediatr 2007;96:1445-9.

33) Hamprecht K, Maschmann J, Muller D, Dietz K, Besenthal I, Goelz R, et al. Cytomegalovirus (CMV) inactivation in breast milk: reassessment of pasteurization and freeze-thawing. Pediatr Res 2004;56:529-35.

34) Yoo HS, Sung SI, Jung YJ, Lee MS, Han YM, Ahn SY, et al. Prevention of cytomegalovirus transmission via breast milk in extremely low birth weight infants. Yonsei Med J 2015;56:9981006.
35) Stock K, Griesmaier E, Brunner B, Neubauer V, Kiechl-Kohlendorfer U, Trawoger R. Pasteurization of breastmilk decreases the rate of postnatally acquired cytomegalovirus infections, but shows a nonsignificant trend to an increased rate of necrotizing enterocolitis in very preterm infants: a preliminary study. Breastfeed Med 2015;10:113-7.

36) Ben-Shoshan M, Mandel D, Lubetzky R, Dollberg S, Mimouni FB. Eradication of cytomegalovirus from human milk by microwave irradiation: a pilot study. Breastfeed Med 2016;11:186-7.

37) Lloyd ML, Hod N, Jayaraman J, Marchant EA, Christen L, Chiang $\mathrm{P}$, et al. Inactivation of cytomegalovirus in breast milk using ultraviolet-C irradiation: opportunities for a new treatment option in breast milk banking. PLoS One 2016;11: e0161116.

38) Lombardi G, Garofoli F, Manzoni P, Stronati M. Breast milkacquired cytomegalovirus infection in very low birth weight infants. J Matern Fetal Neonatal Med 2012;25 Suppl 3:57-62.

39) Fischer C, Meylan P, Bickle Graz M, Gudinchet F, Vaudaux B, Berger C, et al. Severe postnatally acquired cytomegalovirus infection presenting with colitis, pneumonitis and sepsis-like syndrome in an extremely low birthweight infant. Neonatology 2010;97:339-45.

40) Okulu E, Akin IM, Atasay B, Ciftci E, Arsan S, Turmen T. Severe postnatal cytomegalovirus infection with multisystem involvement in an extremely low birth weight infant. J Perinatol 2012;32:72-4.

41) Tran L, Ferris M, Norori J, Stark M, Craver R, Dowd S, et al. Necrotizing enterocolitis and cytomegalovirus infection in a premature infant. Pediatrics 2013;131:e318-22.

42) Kimberlin DW, Jester PM, Sanchez PJ, Ahmed A, Arav-Boger R, Michaels MG, et al. Valganciclovir for symptomatic congenital cytomegalovirus disease. N Engl J Med 2015;372:933-43.

43) Bevot A, Hamprecht K, Krageloh-Mann I, Brosch S, Goelz R, Vollmer B. Long-term outcome in preterm children with human cytomegalovirus infection transmitted via breast milk. Acta Paediatr 2012;101:e167-72.

44) Brecht KF, Goelz R, Bevot A, Krageloh-Mann I, Wilke M, Lidzba K. Postnatal human cytomegalovirus infection in preterm infants has long-term neuropsychological sequelae. J Pediatr 2015;166:834-9.el.

45) Jim WT, Chiu NC, Ho CS, Shu CH, Chang JH, Hung HY, et al. Outcome of preterm infants with postnatal cytomegalovirus infection via breast milk: a two-year prospective follow-up study. Medicine (Baltimore) 2015;94:e1835. 